\title{
PROPERTIES OF INJECTIVE HULLS OF A RING HAVING A COMPATIBLE RING STRUCTURE*
}

\author{
BARBARA L. OSOFSKY** \\ Department of Mathematics, Rutgers University, 110 Frelinghuysen Road, Piscataway, NJ 08904-8019, USA \\ e-mail: osofsky@math.rutgers.edu
}

\author{
JAE KEOL PARK \\ Department of Mathematics, Busan National University, Busan 609-735, South Korea \\ e-mail:jkpark@pusan.ac.kr \\ and S. TARIQ RIZVI \\ Department of Mathematics, Ohio State University, Lima, OH 45804-3576, USA \\ e-mail:rizvi.1@osu.edu
}

\begin{abstract}
If the injective hull $E=E\left(R_{R}\right)$ of a $\operatorname{ring} R$ is a rational extension of $R_{R}$, then $E$ has a unique structure as a ring whose multiplication is compatible with $R$-module multiplication. We give some known examples where such a compatible ring structure exists when $E$ is a not a rational extension of $R_{R}$, and other examples where such a compatible ring structure on $E$ cannot exist. With insights gleaned from these examples, we study compatible ring structures on $E$, especially in the case when $E_{R}$, and hence $R_{R} \subseteq E_{R}$, has finite length. We show that for $R_{R}$ and $E_{R}$ of finite length, if $E_{R}$ has a ring structure compatible with $R$-module multiplication, then $E$ is a quasiFrobenius ring under that ring structure and any two compatible ring structures on $E$ have left regular representations conjugate in $\Lambda=\operatorname{End}_{R}\left(E_{R}\right)$, so the ring structure is unique up to isomorphism. We also show that if $E_{R}$ is of finite length, then $E_{R}$ has a ring structure compatible with its $R$-module structure and this ring structure is unique as a set of left multiplications if and only if $E_{R}$ is a rational extension of $R_{R}$.
\end{abstract}

2002 Mathematics Subject Classification. Primary 16D50, 16S90, Secondary $16 \mathrm{~L} 60$.

1. Introduction. Throughout this paper, all rings are associative with identity, and all modules are unitary. The concepts are standard ones that can be found in books such as [7], [11] or [1].

In the middle of the 20th century, there was a lot of activity in ring theory. The area of interest in this paper arose from a concern with rings of quotients of arbitrary rings, extending the concept of the classical ring of quotients for commutative rings or Öre domains. These rings of quotients of a ring $R$ are rings $S$ that are essential extensions of the ring $R$ as a right $R$-module; that is, they are overrings of $R$ which, as $R$-modules, embed in the injective hull of the module $R_{R}$. The case when the injective hull $E_{R}$ of $R_{R}$ is a rational extension of $R_{R}$, that is, $\operatorname{hom}_{R}(E / R, R)=0$, was studied in papers such as [8], [9] and [10]. In the rational extension case, the map $\lambda: \operatorname{End}_{R}\left(E_{R}\right) \longrightarrow E$,

*To Patrick Smith on his 65 th birthday, with thanks for all he has done for ring theory.

** Corresponding author. 
$\lambda \mapsto \lambda(1)$ is an abelian group isomorphism which induces a ring structure on $E$, making it isomorphic to $\operatorname{End}_{R}\left(E_{R}\right)$. There is an exposition of the maximal rational extension of a ring, with the previous work summarized in [7]. We formally state these early results in one combined theorem.

THEOREM. Let $R$ be a ring with identity $1_{R}$ such that the injective hull $E=E\left(R_{R}\right)$ is a rational extension of $R_{R}$, that is, $\operatorname{hom}_{R}(E / R, R)=0$. Then the map $f \in \operatorname{End}_{R}\left(E_{R}\right) \mapsto$ $f\left(1_{R}\right) \in E$ induces a ring multiplication on $E$, extending the $R$-module multiplication on $E$, that is, the ring multiplication $E \times E \rightarrow E$ restricts to $R$-module multiplication $E \times R \rightarrow E$.

If $R$ is right non-singular, then the ring structure on $E$ makes it a right self-injective von Neumann regular ring.

Early results on the situation when the injective hull $E\left(R_{R}\right)$ is not a rational extension of $R_{R}$ consisted of some examples where the injective hull has a compatible ring structure, and other examples where it does not. See [14], [15] and [16]. Ten years later there was a theorem by Lang (see [12]) that a commutative artinian ring has an injective hull with a compatible ring structure if and only if the ring is quasi-Frobenius. But almost nothing else was known before the turn of the millennium.

2. Crucial preliminaries. NotAtion. The following notation will be used throughout the rest of this paper.

(a) $R$ will denote a ring with identity denoted by $1_{R}$.

(b) A right (respectively left) $R$-module $M$ will often be denoted by $M_{R}$ (respectively ${ }_{R} M$ ).

(c) $E=E_{R}=E\left(R_{R}\right)$ will denote a fixed injective hull of $R_{R}$ with a fixed essential embedding of the right $R$-module $R_{R}$ into $E_{R}$.

(d) $\Lambda$ will denote the ring $\operatorname{End}_{R}\left(E_{R}\right)$.

(e) We use $J(-)$ to denote the Jacobson radical of a ring, and socle(-) to denote the socle of a module. A subscript may be used to indicate the appropriate side; in its absence right is assumed.

(f) The composition length of a module $M$ of finite length will be denoted by $\ell(M)$.

(g) The $n \times n$ matrices over a ring $F$ will be denoted by $\mathcal{M}_{n}(F)$.

(h) Matrix units in $\mathcal{M}_{n}(F)$ will be denoted by the letter $\varepsilon$ with appropriate subscripts; for example, $\varepsilon_{11}=\left[\begin{array}{cc}1 & 0 \\ 0 & 0\end{array}\right]$, where $\mathbf{0}$ is the appropriate matrix all of whose entries are 0 . The letter $\varepsilon$ with other annotations will also be used to indicate some other idempotents.

We say that a ring structure $\mathcal{E}$ on $E\left(R_{R}\right)$ is compatible if the ring multiplication extends the $R$-module scalar multiplication. Rings $R$ such that $E\left(R_{R}\right)$ has a compatible ring multiplication were called (right) Osofsky compatible in [3]. In this paper we omit the word right. In [2] a particular Osofsky compatible ring $R$ with ring structure $\mathcal{E}$ on $E\left(R_{R}\right)$ was studied (Example 7 in this paper) using the left regular representation of $\mathcal{E}$. This turned out to be a very effective way of getting away from the early approach of mainly looking at specific examples of rings to see if they are or not Osofsky compatible. The use of the left regular representation is crucial to the new results in Section 3.2 here. So we formally define the left regular representation (left multiplications) of a 
compatible ring structure on $E_{R}$ and then show in Remarks 1-3 the definition that this indeed is the left regular representation of a compatible ring structure.

Definition (Compatible left multiplications). Let $\lambda: E_{R} \rightarrow \Lambda=\operatorname{End}_{R}\left(E_{R}\right)$ be an additive group monomorphism. Denote $\lambda(u)$ by $u_{\lambda}$, that is, set $\lambda(u)(v)=u_{\lambda}(v)$ to avoid possibly confusing adjacent parentheses. Assume

(i) these left multiplications, $\left\{u_{\lambda}: u \in E\right\}$, are closed under composition in $\Lambda$,

(ii) $\left(1_{R}\right)_{\lambda}=1_{\Lambda}$ and

(iii) $u_{\lambda}\left(1_{R}\right)=u$.

Define the multiplication of the ring structure $\mathcal{E}$ by $\cdot: E \times E \rightarrow E,(u, v) \mapsto$ $u \cdot v=u_{\lambda}(v)$.

We say that $\lambda$ is a left multiplication map on $E_{R}$ with $m_{\lambda}$ left multiplication by $m, \cdot$ is a compatible ring multiplication on $E_{R}$ induced by $\lambda$ and $\mathcal{E}=\langle E,+, \cdot\rangle$ is a compatible ring structure on $E_{R}$. The image of $\lambda$ is called the left regular representation of $\mathcal{E}$ in the literature. We will often refer to the image of a left multiplication map $\lambda$ as $E_{\lambda}$, but when the meaning is clear we will also drop the subscript $\lambda$ and/or the letter $\mathcal{E}$ and just use the letter $E$ to refer to this ring structure.

Now assume we have such a left multiplication map.

REMARK 1 . The image $E_{\lambda}$ of $\lambda$ is a subring of $\Lambda$ since $\lambda$ is additive, $E_{\lambda}$ is closed under composition in $\Lambda$ by $(i)$ and the identity of $\Lambda$ is in the image of $\lambda$ by (ii).

REMARK 2 . The maps $\lambda$ and the evaluation $m_{\lambda} \mapsto m_{\lambda}\left(1_{R}\right)$ are inverse abelian group isomorphisms between $E$ and the image of $\lambda$ by (ii) and (iii).

REMARK 3. For all $u$ and $v$ in $E, u \cdot v=u_{\lambda} \circ v_{\lambda}\left(1_{R}\right)$ since $u_{\lambda}(v)=u_{\lambda}\left(v_{\lambda}\left(1_{R}\right)\right)$ by (iii) and $u_{\lambda}\left(v_{\lambda}\left(1_{R}\right)\right)=u_{\lambda} \circ v_{\lambda}\left(1_{R}\right)$ by definition of composition of functions. Hence, evaluation at $1_{R}$ is a ring isomorphism from $\left\langle E_{\lambda},+, \circ\right\rangle$ to the $\operatorname{ring}\langle E,+, \cdot\rangle$, and its inverse isomorphism is $\lambda$.

REMARK 4. Let $R$ be the ring of upper triangular $2 \times 2$ matrices over a field $F$. Then $E$ is the ring of all $2 \times 2$ matrices and by standard linear algebra

$$
\left[\begin{array}{ll}
a & b \\
c & d
\end{array}\right]_{\lambda}=\left[\begin{array}{llll}
a & 0 & b & 0 \\
0 & a & 0 & b \\
c & 0 & d & 0 \\
0 & c & 0 & d
\end{array}\right]
$$

where

$$
\left[\begin{array}{ll}
a & b \\
c & d
\end{array}\right]_{\lambda}\left(\left[\begin{array}{cc}
x & y \\
z & w
\end{array}\right]\right)=\left[\begin{array}{llll}
a & 0 & b & 0 \\
0 & a & 0 & b \\
c & 0 & d & 0 \\
0 & c & 0 & d
\end{array}\right]\left[\begin{array}{c}
x \\
y \\
z \\
w
\end{array}\right]=\left[\begin{array}{c}
a x+b z \\
a y+b w \\
c x+d z \\
c y+d w
\end{array}\right]
$$

Knowing that $R$ is a subring of $E$ makes it easy to check that this has all of the properties of compatible left multiplications despite the fact that it has a different appearance than the usual multiplication of matrices

$$
\left[\begin{array}{ll}
a & b \\
c & d
\end{array}\right] \cdot\left[\begin{array}{ll}
x & y \\
z & w
\end{array}\right]=\left[\begin{array}{ll}
a x+b z & a y+b w \\
c x+d z & c y+d w
\end{array}\right] .
$$


The following observation indicates why using this definition of a compatible multiplication can be powerful. Without the definition, getting set theoretically distinct ring structures can be quite messy and convey very little insight.

REMARK 5. Let $\phi$ be any automorphism of $E_{R}$. Set

$$
\mathcal{C}=\left\{\phi^{-1} m_{\lambda} \phi: m \in E\right\} .
$$

Then $\mathcal{C}$ is closed under composition and contains the identity. It is a subring of $\Lambda$ and is isomorphic to $E_{\lambda}$ under the inner automorphism of $\Lambda$ induced by conjugation by $\phi$. Moreover, $\left(\phi\left(1_{R}\right)\right)_{\lambda}$ is a unit in $\Lambda$ since $R_{R}$ is essential in $E_{R}$; this follows since $\left(\phi\left(1_{R}\right)\right)_{\lambda} r=\phi(r)$ for all $r \in R$ and $R$ is essential in $E$ so $\left(\phi\left(1_{R}\right)\right)_{\lambda}$ is one-to-one and has essential injective image. Then

$$
\begin{aligned}
0 & =\phi^{-1} m_{\lambda} \phi \Longleftrightarrow 0=\left(\phi^{-1} m_{\lambda} \phi\left(1_{R}\right)\right)_{\lambda} \Longleftrightarrow 0=\phi^{-1} m_{\lambda}\left(\phi\left(1_{R}\right)\right)_{\lambda} \\
& \Longleftrightarrow 0=\phi\left(\phi^{-1} m_{\lambda}\left(\phi\left(1_{R}\right)\right)_{\lambda}\right)\left(\phi\left(1_{R}\right)\right)_{\lambda}^{-1} \Longleftrightarrow m_{\lambda}=0 \Longleftrightarrow m=0,
\end{aligned}
$$

so the map $\lambda^{\prime}: E \rightarrow \mathcal{C}$ can be defined as the inverse to the map $\phi^{-1} m_{\lambda} \phi \mapsto$ $\phi^{-1} m_{\lambda} \phi\left(1_{R}\right) \in E$, and this will give us a ring of left multiplications isomorphic to $E_{\lambda}$ but not equal to it unless $\phi$ commutes with each $m_{\lambda}$.

Note that, in general, $m_{\lambda^{\prime}} \neq \phi^{-1} m_{\lambda} \phi$. Instead, we have $\left(\phi^{-1} m_{\lambda} \phi\left(1_{R}\right)\right)_{\lambda^{\prime}}=\phi^{-1} m_{\lambda} \phi$, where $\phi^{-1} m_{\lambda} \phi\left(1_{R}\right)$ may very well not be equal to $m$.

2.1. Early examples. We now look at some examples that have been around for a long time. All but the first were probably known in the 1960 s although not necessarily published then. These early examples were interesting, but did not appear to yield any significant insight. We just sketch proofs of the properties claimed for these examples as an indication of ideas and techniques that help in placing our new results in context.

EXAMPLE 1 [12]. A commutative artinian ring $R$ has a compatible ring structure on its injective hull if and only if $R$ is quasi-Frobenius.

We will not prove this here, but the next example is a special case of Lang's result which will have a significant generalization in Section 3.2, Theorem 8.

EXAMPLE 2. Let $F$ be a field, and $R$ the $F$-algebra with basis $\{1, x, y\}$ and relations $x^{2}=y^{2}=x y=y x=0$. Then the injective hull of $E=E\left(R_{R}\right)$ of $R$ does not have a compatible ring structure.

Proof. $E_{R}$ is a direct sum $E_{R}=E(x R) \oplus E(y R)$. Let $\varepsilon_{x x}$ and $\varepsilon_{y y}$ be the projections in $\Lambda$ of $E$ onto these summands. Since these are orthogonal idempotents summing to $1_{\Lambda}$, they are the unique projections of the identity of $\Lambda$ with respect to the left direct decomposition $\Lambda=\Lambda \varepsilon_{x x} \oplus \Lambda \varepsilon_{y y}$. If $\mathcal{E}$ is a compatible ring structure on $E$, the identity of $\Lambda$ lies in $\mathcal{E} \subseteq \Lambda, \varepsilon_{x x} E=E(x R)$ and $\varepsilon_{y y} E=E(y R)$, the projections of the identity of $\mathcal{E}$ with respect to the direct sum decomposition $E=\varepsilon_{x x} E \oplus \varepsilon_{y y} E$ must lie in $\mathcal{E} . E(x R)$ and $E(y R)$ are injective hulls of isomorphic simple $R$-modules and so are isomorphic $R$-modules. Hence,

$$
E \cong \operatorname{End}_{E}(E) \cong \mathcal{M}_{2}\left(\operatorname{End}_{E}\left(\varepsilon_{x x} E\right)\right)
$$

has a nilpotent socle of $F$-dimension 4 but the socle of $R$ has $F$-dimension 2, so if such a ring structure existed, some simple submodule of $\mathcal{E}_{R}$ would not intersect $R_{R}$. 
EXAMPLE 3. $R=\left[\begin{array}{cc}\mathbb{H} & \mathbb{H}_{\mathbb{C}} \\ 0 & \mathbb{C}\end{array}\right]$ is an example where the injective hulls on each side are von Neumann regular rational extensions of $R$ because $R$ is non-singular on both sides. They have completely different ring structures since $E\left(R_{R}\right) \cong \mathcal{M}_{3}(\mathbb{C})$ and $E\left({ }_{R} R\right) \cong \mathcal{M}_{2}(\mathbb{M})$.

Our next example will be, if the appropriate dimension is finite, artinian with a Morita duality but not an Artin algebra. We are not sure whether or not it actually appeared in print; it was not in [14] or [15], but it is certainly in the spirit of the early examples. It is not Osofsky compatible in a way fundamentally different than any other example of a ring which is not Osofsky compatible given in this paper. This phenomenon cannot occur for Artin algebras, because it implicitly depends on the fact that $R$ is not finitely generated over its center.

EXAMPLE 4. Let $K$ be a field such that there exists an endomorphism $\sigma$ of $K$ such that the dimension of $K$ over $\sigma(K)$ is $n>1$. For example, one might have $K=\mathbb{R}(x)$ (the quotient field of $\mathbb{R}[x])$ and $\sigma(x)$ the endomorphism induced by $x \mapsto x^{2}$. Set $F=\sigma(K)$, so in our example, $F=\mathbb{R}\left(x^{2}\right)$, and let

$$
\langle R,+\rangle=F \oplus{ }_{\sigma(F)} F_{F},
$$

where $\left.{ }_{(\sigma(F)} F_{F}\right)^{2}=0$. That is, for $\alpha, \beta, \gamma, \delta \in F$,

$$
(\alpha, \beta)(\gamma, \delta)=(\alpha \gamma, \sigma(\alpha) \delta+\beta \gamma)
$$

Then $R$ is not Osofsky compatible.

Proof. Think of $F=\sigma(K)$ as saying $K=\sigma^{-1}(F)$. Replace $F$ by $K$ in the definition of $R$ and map $\left(1_{F}, 0\right)$ to $\left(1_{K}, 0\right)$ to get an $R$-module embedding of $R_{R}$ into the $R$-module $E_{R}=K \oplus_{\sigma(K)} F_{F}$. We now justify this notation. $E_{R}$ is clearly an essential extension of $R_{R}$. The only proper right ideal of $R$ is $\left(0, F_{F}\right)$. Any $R$-map from $\left(0, F_{F}\right)$ to $R_{R}$ or to $E_{R}$ must take $(0,1)$ to $(0, \alpha)$ for some $\alpha \in F$. Since these maps are given by left multiplication by $\left(\sigma^{-1}(\alpha), 0\right)$ in $E$, we observe that

$$
\operatorname{End}_{R}\left(0, F_{F}\right) \cong{ }_{K} K_{\sigma(K)} \cong{ }_{\sigma^{-1}(F)} K_{F},
$$

and $E$ is indeed the right injective hull of $R_{R}$.

Assume that $E_{R}$ has a compatible ring structure. Then $J(E)$ would have to contain the nilpotent (right) ideal $\left(0, F_{F}\right)$ and $E / J(E)$ would have to be isomorphic to a quotient of ${ }_{\sigma^{-1}(F)} K_{F}$ which is a simple $R-R$ bimodule which is not a ring. This contradiction to basic (artinian) ring theory shows that $R$ is not Osofsky compatible.

If $n<\infty, E$ is a finitely generated right $R$-module containing a copy of the only simple right $R$-module, and so a right injective cogenerator. By [13], $\operatorname{hom}_{R}(, E)$ induces a Morita duality between finitely generated right $R$-modules and finitely generated left $\operatorname{End}_{R}(E)$-modules. So Morita duality does not guarantee an Osofsky compatible ring structure on $E$.

We observe that for $R$ the ring in Example 4, the left injective hull $E\left({ }_{R} R\right)$ of $R$ is isomorphic to $E\left(0, \sigma_{(F)} F\right) \cong \prod_{n} F$ and leave to the interested reader the proof that this cannot be given a compatible ring structure. The proof in Example 2 may help in the case $n<\infty$. 
The proof for our next example is essentially the original proof, which appeared in [14] and [15]. There is nothing significant about using $\mathbb{Z}_{4}$ here other than it is a nice concrete ring. A generalization of this example replacing $\mathbb{Z}_{4}$ by an arbitrary local quasi-Frobenius ring $A$ is part of the proof of Theorem 9 in Section 3.2.

EXAmple 5. Let $R=\left[\begin{array}{cc}\mathbb{Z}_{4} & 2 \mathbb{Z}_{4} \\ 0 & \mathbb{Z}_{4}\end{array}\right]$. Then the injective hull of $R$ does not have any compatible ring structure.

Proof. Observe that

$$
\begin{aligned}
E & =E\left(R_{R}\right)=E\left(\left[\begin{array}{cc}
2 \mathbb{Z}_{4} & 0 \\
0 & 0
\end{array}\right]\right) \oplus E\left(\left[\begin{array}{cc}
0 & 2 \mathbb{Z}_{4} \\
0 & 0
\end{array}\right]\right) \oplus E\left(\left[\begin{array}{cc}
0 & 0 \\
0 & \mathbb{Z}_{4}
\end{array}\right]\right) \\
& =\varepsilon_{1} E \oplus \varepsilon_{2} E \oplus \varepsilon_{3} E,
\end{aligned}
$$

where $\left\{\varepsilon_{i}: 1 \leq i \leq 3\right\}$ are the orthogonal idempotent projections in $\Lambda$ with respect to this direct sum decomposition of $E$. Note that $\varepsilon_{2} E$ and $\varepsilon_{3} E$ are isomorphic. Hence, $\varepsilon_{2} E$ must contain an element $x$ of additive order 4 with $2 x=2 \varepsilon_{2}(x)=\left[\begin{array}{cc}0 & 2 \mathbb{Z}_{4} \\ 0 & 0\end{array}\right] \in \varepsilon_{2} E \cap R$. Since $x=\varepsilon_{2}(x)$, the additive order of $\varepsilon_{2}$ must be 4 . On the other hand, $\varepsilon_{2}\left(1_{R}\right) \in \varepsilon_{2} E$ satisfies $2 \varepsilon_{2}\left(1_{R}\right)\left(\left[\begin{array}{cc}0 & 2 \mathbb{Z}_{4} \\ 0 & 0\end{array}\right]\right)=0$.

Now assume that there is a compatible ring structure $E_{\lambda}$ on $E$. Since $\varepsilon_{i} E_{\lambda}$ is a right ideal of $E_{\lambda}$, which is a direct summand of $\left(E_{\lambda}\right)_{E_{\lambda}}$ and $\sum_{i=1}^{3} \varepsilon_{i}\left(1_{R}\right)_{\lambda}=1_{\Lambda},\left\{\varepsilon_{i}\left(1_{R}\right)_{\lambda}\right\}$ are orthogonal idempotent projections of the identity of $E_{\lambda}$ with respect to the given decomposition of $E$, so $\varepsilon_{2}=\varepsilon_{2}\left(1_{R}\right)_{\lambda}$ must have order 4 and $2 \varepsilon_{2}(x)=\left[\begin{array}{cc}0 & 2 \mathbb{Z}_{4} \\ 0 & 0\end{array}\right] \neq 0$, which is a contradiction.

It is not too difficult to show that $\Lambda \cong \mathbb{Z}_{4} \times\left[\begin{array}{ll}\mathbb{Z}_{4} & \mathbb{Z}_{4} \\ \mathbb{Z}_{4} & \mathbb{Z}_{4}\end{array}\right]$ with matrix multiplication on the matrices, and $E_{R} \cong \mathbb{Z}_{4} \times\left[\begin{array}{cc}\mathbb{Z}_{2} & \mathbb{Z}_{4} \\ \mathbb{Z}_{2} & \mathbb{Z}_{4}\end{array}\right]$ with $\varepsilon_{1}=\left(1_{\mathbb{Z}_{4}},\left[\begin{array}{cc}1_{\mathbb{Z}_{2}} & 0 \\ 0 & 0\end{array}\right]\right), \varepsilon_{2}=\left(0_{\mathbb{Z}_{4}},\left[\begin{array}{cc}1_{\mathbb{Z}_{2}} & 0 \\ 0 & 0\end{array}\right]\right)$, $\varepsilon_{3}=\left(0_{\mathbb{Z}_{4}},\left[\begin{array}{cc}0 & 0 \\ 0 & 1 \mathbb{Z}_{4}\end{array}\right]\right)$ the projections of $1_{R}$ in $E$. A difference between this and what occurs in Example 7 is that here the ring we compute is $\Lambda$, whereas in Example 7 it is $\mathcal{E}$.

We next look at an example which is different from most of the other early examples as it is not a small ring but the injective hull is rather obvious. There is a non-commutative version of this example using the example of a one-sided injective cogenerator found in [6]. In that version there is a compatible multiplication on its injective side, but it is not clear whether or not it has such a compatible multiplication on the other side.

EXAMPLE 6 . Let $\mathbb{Z}_{(p)}$ denote the integers localized at the prime $p, \widehat{\mathbb{Z}}_{(p)}$ the $p$-adic integers and $\mathbb{Z}_{p^{\infty}}$ the $p$-torsion subgroup of $\mathbb{Q} / \mathbb{Z}$. Let $R$ be the ring with additive group $\mathbb{Z}_{(p)} \oplus \mathbb{Z}_{p^{\infty}}$ and $\left(\mathbb{Z}_{p^{\infty}}\right)^{2}=0$. Then

$$
E\left(R_{R}\right)=\widehat{\mathbb{Z}}_{(p)} \oplus \mathbb{Z}_{p^{\infty}},
$$

because $\mathbb{Z}_{p^{\infty}}$ is an injective cogenerator for $\mathbb{Z}_{(p)}$ and $\widehat{\mathbb{Z}}_{(p)}$, and $\widehat{\mathbb{Z}}_{(p)}=\operatorname{End}_{\mathbb{Z}_{(p)}}\left(\mathbb{Z}_{p^{\infty}}\right)$. This has a ring structure obtained by setting $\left(\mathbb{Z}_{p^{\infty}}\right)^{2}=0$ and letting $\widehat{\mathbb{Z}}_{(p)}$ act on it on both sides. The compactness of the $p$-adic topology implies that there is only one possible multiplication on $\widehat{\mathbb{Z}}_{(p)}$, so this ring structure is unique. Since any non-zero element 
$x \in \widehat{\mathbb{Z}}_{(p)} \backslash \mathbb{Z}_{(p)}$ has a non-zero map to $\frac{1}{p} \mathbb{Z} \subseteq \mathbb{Z}_{p^{\infty}} . E$ is not a rational extension of $R$. Here $E$ is a self-injective ring.

2.2. Recent examples. Examples such as those in Section 2.1 did not appear to shed much light on whether or not some other ring was Osofsky compatible, or if it was, what properties a compatible ring structure $\mathcal{E}$ on its injective hull might have. But the first two examples in this subsection do shed light on further work in the area.

Our first example looks very much like Example 5 except that matrices which did not form a ring there do form a ring here. This example is due to Birkenmeier, Osofsky, Park, and Rizvi.

ExAmple 7. Let $A$ be a commutative local quasi-Frobenius ring with non-zero Jacobson radical $J$. Set

$$
R=\left[\begin{array}{cc}
A & A / J \\
0 & A / J
\end{array}\right]
$$

Then

(a) $E=E\left(R_{R}\right) \cong A \times\left[\begin{array}{cc}A / J & A / J \\ A / J & A / J\end{array}\right]$ with the embedding $\left[\begin{array}{cc}\alpha & \beta \\ 0 & \delta\end{array}\right] \mapsto \alpha+\left[\begin{array}{cc}\alpha+J & \beta \\ 0 & \delta\end{array}\right]$ has a ring structure $\lambda$ as a quasi-Frobenius ring compatible with the $R$-module structure, and

$$
\operatorname{hom}_{R}(E / R, E) \cong \operatorname{hom}_{R}\left(\left[\begin{array}{ll}
A / J & 0 \\
A / J & 0
\end{array}\right], A\right) \neq 0 .
$$

(b) There exists a compatible ring structure $\lambda^{\prime}$ on $E$ with $E_{\lambda} \neq E_{\lambda^{\prime}}$.

(c) All compatible ring structures on $E$ are isomorphic to $E_{\lambda}$.

(d) $E_{\lambda}$ is also a compatible ring structure on the injective hull of the left regular representation ${ }_{R} R$.

Proof. See [2]. We begin with the left $R$-module ${ }_{R} R$ which is a left projective generator, and apply the $A$-duality $\operatorname{hom}_{A}\left({ }_{-}, A\right)$ to produce a right $R$-injective cogenerator. Just as in the case of vector spaces over a field, the $A$-dual of a matrix is its transpose. That is,

$$
\left[\begin{array}{cc}
A & 0 \\
A / J & A / J
\end{array}\right]_{R}
$$

is an injective cogenerator where the $R$-module multiplication is matrix multiplication.

$\operatorname{Re}(\mathrm{a})$ : The socle of $R$ is [ $\left.\begin{array}{cc}\operatorname{socle}(A) & A / J \\ 0 & A / J\end{array}\right]$, which is a direct sum of three simples. Then the injective hull of $R_{R}$ is

$$
\begin{aligned}
E\left(R_{R}\right) & \cong\left[\begin{array}{cc}
A & 0 \\
0 & 0
\end{array}\right] \bigoplus\left[\begin{array}{cc}
A / J & A / J \\
0 & 0
\end{array}\right] \bigoplus\left[\begin{array}{cc}
0 & 0 \\
A / J & A / J
\end{array}\right] \\
& \cong A \times\left[\begin{array}{ll}
A / J & A / J \\
A / J & A / J
\end{array}\right],
\end{aligned}
$$

where the matrices clearly form a ring and the bottom line is clearly a ring direct product. It is an easy check that the product ring structure is compatible with the given embedding of $R$ into $E$, and $\operatorname{hom}_{R}(E / R, E)$ is the direct sum of the composition factors of $E / R$ isomorphic to [ $\left[\begin{array}{cc}\operatorname{socle}(A) & 0 \\ 0 & 0\end{array}\right] \subseteq R$. 
$\operatorname{Re}(b)$ : We use Remark 5. The maps $\phi_{\mu_{1} \mu_{2}}:\left(1-\varepsilon_{11}\right) \mapsto\left(1-\varepsilon_{11}\right), \varepsilon_{11} \mapsto \varepsilon_{11}+$ $\left(1-\varepsilon_{11}\right) \mu_{1}, \varepsilon_{21} \mapsto \varepsilon_{21}+\left(1-\varepsilon_{11}\right) \mu_{2}$ for $\mu_{1}, \mu_{2} \in \operatorname{socle}(A)$ generate automorphisms of $E$, and these are all distinct since

$$
\begin{aligned}
\phi_{\mu_{1}, \mu_{2}}^{-1}\left(\varepsilon_{i 1}\right)_{\lambda} \phi_{\mu_{1}, \mu_{2}}\left(\varepsilon_{11}\right) & =\phi_{\mu_{1}, \mu_{2}}^{-1}\left(\varepsilon_{i 1}\right)_{\lambda}\left(\varepsilon_{11}+\left(1-\varepsilon_{11}\right) \mu_{i}\right) \\
& =\phi_{\mu_{1}, \mu_{2}}^{-1}\left(\varepsilon_{i 1}\right)=\varepsilon_{i 1}+\left(1-\varepsilon_{11}\right) \mu_{i}
\end{aligned}
$$

$\operatorname{Re}(\mathrm{c})$ : Every ring structure on $E$ must arise as in Proposition 3 by careful observation.

$\operatorname{Re}(\mathrm{d})$ : This is just an observation.

Proposition 5 in Section 3 gives an alternate way of getting the form of $E_{R}$ in Example 7 because $\left[\begin{array}{ll}0 & 0 \\ 0 & 1\end{array}\right] R$ is a projective simple $R$-module and $R$ has finite Goldie dimension. This alternate proof illustrates a difference between the rings of Examples 5 and 7. We next use this alternate idea to produce an example modifying Example 3 and showing that the crucial property of Example 2 is that there is only one isomorphism class of simple right modules.

EXAMPLE 8. Let $\left[\mathbb{H}_{,} \mathbb{H}_{\mathbb{H}}\right]$ be the ring whose nilpotent radical is $\left[0, \mathbb{H}_{\mathbb{H}} \mathbb{H}_{\mathbb{H}}\right]$ where $\mathbb{H}$ is the real quarternions, and let

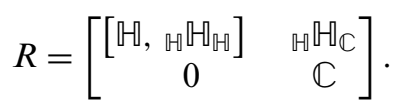

Then $R$ is Osofsky compatible, with

$$
E_{R}=\left[\mathbb{H}, \mathbb{\sharp} \mathbb{H}_{\mathbb{H}}\right] \times \mathcal{M}_{3}(\mathbb{C}),
$$

where

$$
1_{R} \mapsto\left(\left[\begin{array}{ll}
1, & 0
\end{array}\right],\left[\begin{array}{lll}
1 & 0 & 0 \\
0 & 1 & 0 \\
0 & 0 & 1
\end{array}\right]\right)
$$

Proof. The $3 \times 3$ matrices are the injective hull of the second column of $R$ by elementary linear algebra as embodied in Proposition 5 in Section 3. The first factor is

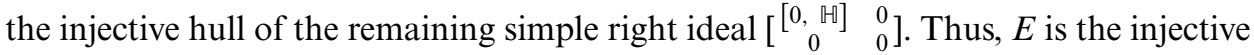
hull of $R_{R}$, and it clearly has a ring structure as a product of quasi-Frobenius rings. One easily checks that the image of $R$ is a subring of $E$ with this direct product structure, and all of the other properties found in Example 7.

However, there is one difference between Example 7 and this example. The primitive idempotent $\varepsilon=\left[\begin{array}{ll}1 & 0 \\ 0 & 0\end{array}\right] \in R$ is a sum $\varepsilon_{\mathbb{}}+\varepsilon_{\mathbb{C}}$ of idempotents in homogeneous components of $E$ where $\varepsilon_{\mathbb{C}}$ is not primitive in $E$.

Example 7 shows some significant properties that can occur. The next examples indicate that there are no comparable results in the general case. Indeed, as Camillo, Herzog, and Nielsen show in [5], $E\left(R_{R}\right)$ need not be a self-injective ring, and it may have non-isomorphic compatible ring structures. 
ExAMPLE 9. Let $F$ be a field, and $V$ an infinite dimensional vector space with a non-degenerate (and symmetric, if you wish $R$ to be commutative) bilinear form $\left.\left\langle{ }_{-},\right\rangle_{-}\right\rangle$on it. Let $R$ have additive group

$$
\langle R,+\rangle=F \oplus V \oplus F,
$$

where $(a, b, c)(\alpha, \beta, \gamma)=(a \alpha, a \beta+b \alpha, a \gamma+c \alpha+\langle b, \beta\rangle)$. Then

$$
E=E\left(R_{R}\right)=(F, \operatorname{hom}(V, F), F),
$$

with hom $(V, F) \otimes V \rightarrow F$ being the dual map. One can make $E$ into a ring by taking a complement $W$ of $V$ in hom $(V, F)$ and setting hom $(V, F) \otimes W \rightarrow F$ any map you wish. For example, it may be zero, and hence, the corresponding ring structure is not commutative, or we may pick a basis for $W$ and make also it a dual basis, so the corresponding ring structure is commutative.

See [5] for additional details.

Our last example shows that, unlike in Example 7, Osofsky compatibility is not a right-left symmetric concept. This is hardly surprising given Example 3.

EXAMPLE 10. Let $\mathbb{Q}$ be the rational numbers, and set $S_{i}=\left[\begin{array}{cc}\varepsilon_{11}^{(i)} \mathbb{Q} & \varepsilon_{12}^{(i)} \mathbb{Q} \\ 0 & 0\end{array}\right]$ for $i=$ $0,1,2, \ldots$. Set

$$
R=\oplus_{i=0}^{\infty} S_{i} \oplus 1 \cdot \mathbb{Q} \subseteq \prod_{i=0}^{\infty} \mathcal{M}_{2}(\mathbb{Q})
$$

Then

(a) $E\left({ }_{R} R\right)=\prod_{i=0}^{\infty} \mathcal{M}_{2}(\mathbb{Q})$ is a rational extension of ${ }_{R} R$.

(b) $E\left(R_{R}\right)$ does not have a compatible ring structure.

Proof. We exploit the fact that a row in a matrix over a field is a right ideal of that matrix ring, but just a vector space over the field on the left.

$\operatorname{Re}(\mathrm{a})$ : Since $S_{i} \oplus 1 \mathbb{Q}$ looks like upper triangular matrices on the left with left annihilators of elements 0 or of the form $R \sum_{\text {finite }} \varepsilon_{11}^{(i)}$ or $R\left(1-\sum_{\text {finite }} \varepsilon_{11}^{(i)}\right)$, the injective hull of $S_{i}$ will be the rational extension $\mathcal{M}_{2}(\mathbb{Q})$, and $E\left({ }_{R} R\right)$ will be isomorphic to the self-injective von Neumann regular ring $\prod_{i=0}^{\infty} \mathcal{M}_{2}(\mathbb{Q})$.

$\operatorname{Re}(\mathrm{b})$ : We note that $J(R)=\operatorname{socle}\left(R_{R}\right)=\bigoplus_{i=0}^{\infty}\left[\begin{array}{cc}0 & \varepsilon_{12}^{(i)} \mathbb{Q} \\ 0 & 0\end{array}\right]$ since every $\varepsilon_{11}^{(i)}$ annihilates every $\left[\begin{array}{cc}0 & \varepsilon_{12}^{(k)} \mathbb{Q} \\ 0 & 0\end{array}\right]$ on the right, this socle is essential in $R_{R}$, and $R_{R} / \bigoplus_{i=0}^{\infty}\left[\begin{array}{cc}0 & \varepsilon_{12}^{(i)} \mathbb{Q} \\ 0 & 0\end{array}\right]$ is von Neumann regular. If there were a compatible ring structure on $E, E / J(E)$ would have to be the full linear ring on this socle. But the biendomorphism ring of the semi-simple left $\Lambda$-module $\bigoplus_{i=0}^{\infty}\left[\begin{array}{cc}0 & \varepsilon_{12}^{(i)} \mathbb{Q} \\ 0 & 0\end{array}\right]$ is the field $\mathbb{Q}$, not the full linear ring $E / J(E)$ which does not have any quotient isomorphic to $\mathbb{Q}$. Then $\bigoplus_{i=0}^{\infty}\left[\begin{array}{cc}0 & \varepsilon_{12}^{(i)} \mathbb{Q} \\ 0 & 0\end{array}\right]$ cannot be a $\Lambda-\Lambda$ bimodule. This contradiction shows that $E\left(R_{R}\right)$ cannot have a compatible ring structure.

3. Main new results. Because of the Camillo-Herzog-Nielsen Example 9, a compatible ring structure $\mathcal{E}$ on $E\left(R_{R}\right)$ need not be self-injective nor unique up to isomorphism. Considerably weaker properties may hold in general, such as Proposition 1(d) 
and other propositions in Section 3.1. Here we use the results in Section 3.1 to show in Section 3.2 that for rings $R$ such that $E_{R}$, and hence, its submodule $R_{R}$, has a composition series, the strong properties of injectivity and uniqueness up to isomorphism do hold if $R$ is Osofsky compatible, and set theoretic uniqueness of $\mathcal{E}$ corresponds to set theoretic uniqueness of injective hulls in $E$ of submodules of $R_{R}$.

3.1. Some more general results. Our first proposition introduces a framework for discussion and notation that will be used in the rest of the paper. Much of it appears several times in the early literature. Item (a) of Proposition 1 shows rather precisely why the situation when $E$ is not a rational extension of $R$ is so hard to study. Item (a) says that there is no way to get a ring structure on $E$ as a quotient ring of $\Lambda$ unless $E$ is a rational extension of $R$. The notation, especially $\mathfrak{I}$, as well as the statements, will be useful in the following.

Proposition 1. Let $R$ be an Osofsky compatible ring, and let $E_{\lambda}$ be a compatible ring structure on E. Set

$$
\mathfrak{I}=\left\{\mu \in \Lambda: \mu\left(1_{R}\right)=0\right\} .
$$

Then, the following hold.

(a) $\mathfrak{I}$ is a left ideal of $\Lambda$. It is a right ideal if and only if $\mathfrak{I}=0$. In particular, $\Lambda / \mathfrak{I}$ is a ring homomorphic image of $\Lambda$ if and only if $\mathfrak{I}=0$.

(b) As left $E_{\lambda}$-modules, $\langle\Lambda ;+\rangle=\langle\mathfrak{I} ;+\rangle \oplus\left\langle E_{\lambda} ;+\right\rangle$.

(c) $\mathfrak{I} \subseteq J(\Lambda)$.

(d) $E_{\lambda} / J\left(E_{\lambda}\right) \cong \Lambda / J(\Lambda)$ which is a right self-injective von Neumann regular ring.

Proof. $\operatorname{Re}$ (a): Let $\mu \in \Lambda$ and $\eta \in \mathfrak{I}$. Then $\mu \eta\left(1_{R}\right)=0$, so $\mathfrak{I}$ is a left ideal. If $\eta \neq 0$, since $E$ is essential over $R_{R}$, there exists an $m \in E$ such that $0 \neq \eta(m) \in R$. The map $1 \mapsto m$ from $R$ to $\Lambda$ extends to a map $v \in \Lambda$ such that $\eta v \notin \mathfrak{I}$. This result does not use the hypothesis that there is a compatible ring structure.

Re (b): For $\mu \in \Lambda$, set $\mu(1)=m \in E$. Then $\mu-m_{\lambda} \in \mathfrak{I}$. Thus, $\Lambda=E_{\lambda}+\mathfrak{I}$. If $m \neq 0, m_{\lambda} \notin \mathfrak{I}$, so the sum is direct.

$\operatorname{Re}(\mathrm{c})$ : Note that $\mathfrak{I} \subseteq J(\Lambda)$ since for all $\lambda$ in the left ideal $\mathfrak{I}, 1+\lambda$ fixes $1_{R}$ and so has zero kernel and essential image, so it must be an isomorphism. Or one could quote the well-known result (see [7]) that the Jacobson radical of the endomorphism ring of an injective module is the set of all endomorphisms with essential kernels. Our proof is just a portion of the standard proof.

$\operatorname{Re}\left(\right.$ d): As left ideals, $\Lambda / J(\Lambda) \cong \Lambda / \mathfrak{I} / J(\Lambda) / \mathfrak{I} \cong E_{\lambda} / J(\Lambda) \cap E_{\lambda} \cong E_{\lambda} / J\left(E_{\lambda}\right)$, where we have used the three previous statements in this proposition plus basic isomorphism theorems. Note that the left ideal being factored out in all but the second quotient is actually two-sided, so the first and last quotients are actually quotient rings, not just quotient left ideals.

Reference [17] shows that $\Lambda / J(\Lambda)$ is a right self-injective von Neumann regular ring and orthogonal idempotents lift orthogonally modulo $J(\Lambda)$. By Proposition 1(c) $E_{\lambda} / J\left(E_{\lambda}\right)$ must also be a right self-injective von Neumann regular ring, and the proof of lifting orthogonal idempotents in [17] goes through almost word for word to show that the property also holds for $E_{\lambda} / J\left(E_{\lambda}\right)$. 
We are now ready for useful new results not requiring chain conditions on $R_{R}$. Here is where almost all of the hard work proving the theorems in the next section is done.

Proposition 2 (Duals of simples are simple). Let $R$ be a ring which is an essential extension on the right of a finitely generated socle. Assume $E$ has a compatible ring structure, and call the corresponding left multiplication ring $E_{\lambda}$ just $E$, omitting the subscript $\lambda$ when it is not needed. Then $E$ has the property that the $E$-dual $\operatorname{Hom}_{E}(S, E)$ is simple for every simple right or left $E$-module $S$.

Proof. We begin with some well-known facts stated with sketches of proof as required details are available in standard texts in the area such as [1].

Since $E_{R}$ is an essential extension of the finite-length socle of $R, E_{R}$ is a direct sum $\bigoplus_{i=1}^{n} E\left(S_{i}\right)$ where $S_{i}$ are simple submodules of $R_{R}$.

The identity $1_{R}$ is a sum $\sum_{i=1}^{n} e_{i}$, where $e_{i} \in E\left(S_{i}\right)$. Since $\left(1_{R}\right)_{\lambda}=1_{\Lambda}, \mathfrak{P}=$ $\left\{e_{i}: 1 \leq i \leq n\right\}$ form a complete set of orthogonal idempotents in $E$. They are primitive since the socle of $E\left(S_{i}\right)$ is essential and uniform, and $E\left(S_{i}\right)=e_{i} E$. Moreover, every non-zero $E$-submodule of $e_{i} E$ must contain $S_{i}$, so the intersection of all the non-zero $E$-submodules of $e_{i} E$ must be $S_{i} E$, and thus $S_{i} E$ is an essential simple submodule of $e_{i} E$.

$\Lambda / J(\Lambda)$ and hence $E / J(E)$ is semi-simple artinian, so it must be a direct product

$$
E / J(E) \cong \prod_{\nu=1}^{k} \mathcal{M}_{\eta_{v}}\left(\Delta_{\nu}\right)
$$

of matrices over division rings $\Delta_{v}$. Each matrix ring has one isomorphism class of simple modules, so there are precisely $k$ isomorphism classes of simple $E$-modules.

Now $e_{i} E \cong e_{j} E$ if and only if $S_{i} \cong S_{j}$ by uniqueness of injective hulls, and $e_{i} E / e_{i} J(E) \cong e_{j} E / e_{j} J(E)$ if and only if $e_{i} E \cong e_{j} E$ by uniqueness of projective covers. Hence, $\operatorname{socle}\left(e_{i} E\right)=S_{i}$ is isomorphic to $\operatorname{socle}\left(e_{j} E\right)=S_{j}$ if and only if the tops $e_{i} E / e_{i} J(E) \cong e_{j} E / e_{j} J(E)$. For a given isomorphism class $\left[e_{i} E\right]$ of right ideals of $E$ generated by a primitive idempotent $e_{i}$, denote this class by $\left[e_{i}\right]$; denote by $\left[e_{\sigma(i)}\right]$ the unique isomorphism class such that $S_{i} \cong e_{\sigma(i)} E / e_{\sigma(i)} J(E)$. To summarize this notation,

$$
\begin{array}{cc}
\operatorname{socle}\left(e_{i} R\right)=S_{i}, & \operatorname{top}\left(e_{i} R\right)=S_{\sigma^{-1}(i),} \\
\operatorname{top}\left(e_{\sigma(i)}\right)=S_{i}, & \operatorname{socle}\left(e_{\sigma(i)}\right)=S_{\sigma(i)} .
\end{array}
$$

Since there are $k$ classes $\left[e_{i}\right]$, by the pigeon hole principle $\sigma$ induces a permutation of the isomorphism equivalence classes of primitive idempotents.

Two minimal right or left ideals of $E / J(E)$ are isomorphic if and only if they lie in the same $\mathcal{M}_{\eta_{v}}\left(\Delta_{v}\right)$, so for primitive idempotents $e, e^{\prime} \in E$,

$$
\begin{aligned}
e E & \cong e^{\prime} E \Longleftrightarrow e E / e J(E) \cong e^{\prime} E / e^{\prime} J(E) \\
& \Longleftrightarrow E e / J(E) e \cong E e^{\prime} / J(E) e^{\prime} \Longleftrightarrow E e \cong E e^{\prime} .
\end{aligned}
$$

We note that the dual $\operatorname{hom}_{E}\left(e_{i} E / e_{i} J(E), \quad E_{E}\right) \cong \operatorname{socle}\left(E_{E}\right) e_{i}$, which is the annihilator of $e_{i} J(E)+\left(1-e_{i}\right) E$ and the left-sided version of this also holds.

Now let $s_{i} \in S_{i}$. Since the idempotents in $\mathfrak{P}$ are primitive and orthogonal and $s_{i} R$ is simple, there is a unique element $e_{\sigma(i)} \in \mathfrak{P}$ such that $e_{i} s_{i} e_{\sigma(i)} \neq 0$. Here the uniqueness is actually as elements, not just isomorphism classes as in $(* *)$. 
It is well known that, for $M_{R}$ a quasi-injective $R$-module, any finitely generated $\operatorname{End}_{R}\left(M_{R}\right)$-submodule of $\operatorname{End}_{R}\left(M_{R}\right) M_{R}$ is its own double annihilator. See for example [7, p. 26]. The right annihilator of $\Lambda e_{i} s_{i}$ in $R$ is a maximal right ideal of $R$. Hence, its annihilator in $E, \Lambda e_{i} s_{i}$, cannot have any non-zero proper cyclic submodules. That is, $\Lambda e_{i} s_{i}$ is a simple $\Lambda$-module. Since $\Im e_{i} s_{i}=0, \Lambda e_{i} s_{i}=E e_{i} s_{i}$ is simple. Then $E e_{i} s_{i}$ must be isomorphic to $E e_{i} / J(E) e_{i}$. On the other hand, $e_{\sigma(i)} J(E)$ annihilates $s_{i}$, so the right annihilator of $s_{i}$ in $E$ is $e_{\sigma(i)} J(E)+\left(1-e_{\sigma(i)}\right)$, and thus, the simple $E$-modules $E e_{i} / J(E) e_{i} \cong E s_{i}$ and are $E$-duals of each other, and every simple right $E$-module is isomorphic to some $e_{\sigma(i)} E / e_{\sigma(i)} J(E)$.

Proposition 3. Assume E has a compatible ring structure $E_{\lambda}$. Let $M_{R}$ be a submodule of $R_{R}$ such that $M$ has two distinct injective hulls $E E$ and $E$ in $E$, where e is an idempotent in $E$ and $e \mapsto f$ induces an isomorphism from $e E$ to $f E$. Then the ring structure on $E$ is not set theoretically unique. This will be the case if there is an idempotent $e^{\prime} \in E \backslash R$ with $[e] \neq\left[e^{\prime}\right], e^{\prime} J(E) \neq 0$ and $\operatorname{socle}(e E) \cong e^{\prime} E / e^{\prime} J(E)$ is simple.

Proof. Let $\phi \in \Lambda$ map $e E$ isomorphically to $f E$ taking $e$ to $f$ and also map $1-e$ to itself. Then $\phi$ is an automorphism of $E_{R}$ since it is the identity on the essential submodule $(e E \cap f E) \oplus(1-e) E$, so by Remark 5,

$$
\mathcal{C}=\left\{\phi^{-1} m_{\lambda} \phi: m \in E\right\}
$$

is the image of a compatible ring structure $\lambda^{\prime}$ on $E$ with $\lambda^{\prime}\left(\phi^{-1} m_{\lambda} \phi(1)\right)=\phi^{-1} m_{\lambda} \phi$. Since $f \notin e E$, ef $\neq f$. Then, since

$$
\phi^{-1} e_{\lambda} \phi(1)=\phi^{-1}\left(e_{\lambda}(f+(1-e))\right)=\phi^{-1}(e f) \neq \phi^{-1}(f)=e,
$$

and $\quad \phi^{-1} e_{\lambda} \phi(1)_{\lambda^{\prime}}=\phi^{-1} e_{\lambda} \phi \in \Lambda$, so $\quad \phi^{-1} e_{\lambda} \phi(1)_{\lambda^{\prime}}\left(\phi^{-1} e_{\lambda} \phi(1)\right)=\phi^{-1} e_{\lambda} \phi(1)=\mathfrak{e} \quad$ is idempotent under the ring structure $\lambda^{\prime}$. In $E_{\lambda}$ there is only one projection of the identity to $e E$ with kernel $(1-e) E$ and that is $e$, so $\mathfrak{e}$ cannot be idempotent in $E_{\lambda}$.

Now let $e^{\prime} \in E \backslash R$ be a primitive idempotent $\notin[e]$ such that the socle of $e E$ is isomorphic to the simple top $e^{\prime} E / e^{\prime} J(E)$ of $e^{\prime} E$ and $e^{\prime} J(E) \neq 0$. Let $s \in \operatorname{socle}(e E), s e^{\prime} \neq 0$. Then $e^{\prime} E$ and $\left(e^{\prime}+s e^{\prime}\right) E$ are distinct injective hulls of $e^{\prime} E \cap\left(e^{\prime}+s e^{\prime}\right) E=e^{\prime} J(E)$.

We can take the previous proposition even further.

Proposition 4. Let $R$ be a ring of finite right Goldie dimension. Let $E_{\lambda}$ and $E_{\lambda^{\prime}}$ be compatible ring structures on $E$. Then there is an automorphism $\phi$ in $\Lambda$ such that $E_{\lambda}$ and $\phi^{-1} E_{\lambda^{\prime}} \phi$ have a complete set $\mathfrak{P}$ of primitive orthogonal idempotents of $\Lambda$ in common.

Proof. Let $R$ be essential over $\oplus_{i=1}^{n} S_{i}$, where the $S_{i}$ are uniform $R$-modules. Let $E_{\lambda}\left(S_{i}\right)$ (respectively $E_{\lambda^{\prime}}\left(S_{i}\right)$ ) denote an injective hull of $S_{i}$ in $E_{\lambda}$ (respectively $E_{\lambda^{\prime}}$ ), and $e_{i}$ (respectively $e_{i}^{\prime}$ ) the projection of the identity to $E_{\lambda}\left(S_{i}\right)$ (respectively $E_{\lambda^{\prime}}\left(S_{i}\right)$ ). Then there is a $\phi$ in $\Lambda$ which maps each $E_{\lambda^{\prime}}\left(S_{i}\right)$ isomorphically onto $E_{\lambda}\left(S_{i}\right)$. The morphism $\phi$ is an automorphism since it is monic on $\oplus_{i=1}^{n} S_{i}$ which is essential, and onto since its image is injective and essential. Set $E_{\mu}=\phi^{-1} E_{\lambda^{\prime}} \phi$. Now for each $i, \phi^{-1} e_{i}^{\prime} \phi\left(e_{i}\right)=\phi^{-1} \phi\left(e_{i}\right)=e_{i}$ since $e_{i}^{\prime}$ acts as the identity on $\phi\left(e_{i} E\right)$. That is, each $e_{i}$ is in $E_{\mu}$ so the $\mathfrak{P}=\left\{e_{i}\right\}$ is a complete set of orthogonal primitive idempotents in both $E_{\lambda}$ and $E_{\mu}$.

The following proposition can be used to get an alternate proof of the form of the injective hull in the Birkenmeier-Osofsky-Park-Rizvi Example 7, and was used in the proof of Example 8. The heart of the proof is in [10]. 
Proposition 5. Let $R$ be a ring, and let $S_{R}$ be a projective simple right ideal of $R$. Let $H=\sum_{S^{\prime} \cong S} S^{\prime}$ be the right ideal of $R$ generated by all right ideals isomorphic to $S$. Let $h$ be an idempotent in $\Lambda$ projecting $E(R)$ to $E(H)$. Then $h \Lambda(1-h)=0$ and $h E=E\left(h \Lambda h_{h R h}\right)$ is a full linear ring over $\operatorname{End}_{R}\left(S_{R}\right)$. Moreover, if $H$ is finitely generated, $\operatorname{hom}_{R}(h \Lambda,(1-h) \Lambda)=0$, so $\operatorname{End}_{R}\left(E\left(H_{R}\right)\right)$ is a ring direct factor of $\Lambda$.

Proof. Note $H \neq 0$ since $S \neq 0$. Now $H$ and hence $h E$ is non-singular since any non-zero element in $H$ has annihilator a direct summand of $R$ distinct from $R$. Since $h^{2}=h, \operatorname{End}_{R}(h E) \cong h \Lambda h$. By non-singularity, the map $h \Lambda h \rightarrow h E, h \lambda h \mapsto h \lambda h\left(1_{R}\right)$ is an abelian group isomorphism, which makes $h E$ into a ring isomorphic to the full linear ring on the semi-simple essential socle $H$ of $h E$.

Let $u \in(1-h) E$. Assume there exists a non-zero $R$-map $\phi$ from $u R$ to $h E$. Since $h E$ is essential over its socle, there is an $r \in R$ with $0 \neq \phi(u) r \in S^{\prime} \cong S$. But $S$ and therefore $S^{\prime}$ is projective, so $\phi: u r R \mapsto S^{\prime}$ splits. Then $(1-h) E$ contains a copy of $S$, contradicting the assumption that $h E$ is essential over the sum of all simple right ideas of $R$ isomorphic to $S$. Thus, $h \Lambda(1-h) E=0$, so $h \Lambda(1-h)=0$. In particular, $h \Lambda=h \Lambda h$. The Peirce decomposition of $\Lambda$ with respect to the ordered idempotents $\{1-h, h\}$ is then, as a block matrix,

$$
\Lambda \cong\left[\begin{array}{c|c}
(1-h) \Lambda(1-h) & (1-h) \Lambda h \\
\hline \mathbf{0} & h \Lambda h
\end{array}\right] .
$$

If $H$ is finitely generated, the only non-zero quotients of $h \Lambda h$ are direct sums of copies of $S$, so there are no such non-zero quotients in $(1-h) E$ and $(1-h) \Lambda h=0$, and this means $\operatorname{End}_{R}\left(E\left(H_{R}\right)\right)$ is a ring direct factor of $\Lambda$.

3.2. Our major theorems given composition series. Example 9 of Camillo, Herzog, and Nielsen shows that, in general, one cannot show properties of a compatible ring structure on the injective hull of a ring comparable to the properties in Example 7. What Example 7 has that Example 9 does not is modules of finite composition length. So our major theorems here will be about rings where the injective hull has a compatible ring structure $E_{\lambda}$, and all modules looked at have finite length. If we are looking at the ring structure on $E$ itself as opposed to the left multiplications, we will drop the subscript $\lambda$. Note that $\ell\left(E_{R}\right) \geq \ell\left(E_{E}\right)$ and $R_{R}$ embeds in $E_{R}$, so $\ell\left(E_{R}\right)<\infty$ implies $\ell\left(R_{R}\right)$ and $\ell\left(E_{E}\right)$ are both finite.

THEOREM 6. Let $E_{R}$ have finite length, and let $E_{R}$ have a compatible ring structure $E_{\lambda}$. Then $E_{\lambda}$ is quasi-Frobenius.

Proof. Given Proposition 2 this is in [13]. The proof is also provided in later texts such as [1]. With duals of simples being simple on both sides, a composition series on one side gives rise to a composition series of annihilators on the other side, so we do not have to impose chain conditions on the left.

THEOREM 7. Let $E_{R}$ have finite length, and let $E_{\lambda}$ and $E_{\lambda^{\prime}}$ be compatible ring structures on $E$. Then $E_{\lambda}$ is conjugate to $E_{\lambda^{\prime}}$ in $\Lambda$.

Proof. By Proposition 4, there is an $E_{\mu}$ conjugate to $E_{\lambda^{\prime}}$ having a complete set $\mathcal{P}=\left\{e_{i}\right\}$ of primitive orthogonal idempotents in common with $E_{\lambda}$. These $\left\{e_{i}\right\} \subseteq \Lambda$ are projections of the identity with respect to the direct sum decomposition ${ }_{\Lambda} E=$ 
$\bigoplus_{i=1}^{n} e_{i} E_{R}$ in all of $\Lambda, E_{\lambda}$ and $E_{\mu}$. By Theorem $6_{E} E$ is left artinian, so ${ }_{\Lambda} E$ is artinian by Proposition 1 . We claim that $\lambda=\mu$.

Take a left composition series for $E$, say

$$
0=C_{0} \subset C_{1} \subset \cdots \subset C_{m} \subset \cdots \subset C_{\left.\ell{ }_{\Lambda} E\right)}=E .
$$

Now assume $\left.\lambda\right|_{C m}=\left.\mu\right|_{C_{m}}$ for some $m<\ell\left({ }_{\Lambda} E\right)$, where the vertical bar denotes function restriction. $C_{0}$ clearly satisfies this. Then for $x \in C_{m+1} \backslash C_{m}$, there is an $e_{i} \in \mathfrak{P}$ such that $e_{i} x=x$ and $J(\widetilde{E}) e_{i} x \subseteq C_{m}$ and $C_{m+1}=\widetilde{E} e_{i} x+C_{m}$, where $\widetilde{E}$ is any one of $\Lambda, E_{\mu}$, $E_{\lambda}$.

Since $\lambda(x)=\lambda\left(e_{i} x\right)=\lambda\left(e_{i}\right) \lambda(x)=e_{i} \lambda(x)$ and similarly $\mu(x)=e_{i} \mu(x), e_{i}(\lambda(x)-$ $\mu(x))=0$. But $\left(1-e_{i}\right) \lambda(x)=\left(1-e_{i}\right) \mu(x)=0$, so $\lambda(x)-\mu(x)=\left(1-e_{i}+e_{i}\right)(\lambda(x)-$ $\mu(x))=0$. By induction, $\left.\lambda\right|_{C_{\ell(E)}}=\left.\mu\right|_{C_{\ell(E)}}$, so $\lambda=\mu$.

Without finite length, Example 9 shows we can never hope to start from 0 and get to all of $E$ in this manner.

The next theorem is a generalization of Lang's theorem, Example 1. It is the only characterization of Osofsky compatible rings of a specific type that appears in this section, and the second that we know of in the literature. The proof indicates how, in the artinian case, one does not lose generality by working with the basic ring Morita equivalent to $R$, even though Osofsky compatibility is not defined by a categorical property.

THEOREM 8. Let $E_{R}$ have finite length. Assume every simple in the socle of $R_{R}$ is in the same isomorphism class $[\widetilde{S}]$ where $\widetilde{S}$ is a simple $R$-module isomorphic to $\varepsilon^{\prime} R / \varepsilon^{\prime} J(R)$, where $\varepsilon^{\prime}$ is a primitive idempotent in $R$. Let $\varepsilon$ be the (unique) idempotent central in $R / J(R)$ such that $\varepsilon R=\sum_{\varepsilon^{\prime \prime} \in R, \varepsilon^{\prime \prime} R \cong \varepsilon^{\prime} R} \varepsilon^{\prime \prime} R$. Then the following are equivalent.

(i) $E_{R}$ has a compatible ring structure.

(ii) $E_{R}$ is a rational extension of $R_{R}$.

(iii) $R_{R}$ is essential over a right ideal of the form $\bigoplus_{i=1}^{k} M_{i}$, where each $M_{i}$ with $i<k$ is isomorphic to $\varepsilon^{\prime} R$ and $M_{k}=\varepsilon R$, and $\varepsilon R \varepsilon$ is quasi-Frobenius.

Proof. Note that $\varepsilon$ acts as a two-sided identity on the simple module $\widetilde{S}$.

$\operatorname{Re}$ (iii) $\Longrightarrow$ (ii): By projecting onto $(1-\varepsilon) R$, without loss of generality we may assume that $(1-\varepsilon) M_{i}=M_{i}$ for $1 \leq i<k$. Let $\left\{\varepsilon_{i, i}: 1 \leq i \leq k\right\}$ be a set of orthogonal idempotents in $\Lambda$ summing to 1 where $\varepsilon_{i, i} E$ is essential over $M_{i}$. Let $R^{\prime}$ be the subring of $R$ generated by $M_{i} \varepsilon$ and the identity. Then the Peirce decomposition of $R^{\prime}$ is

$$
R^{\prime} \cong\left\{\left[\begin{array}{ccc|c}
\varepsilon_{1,1} r & 0 & 0 & \left(M_{1}\right) \varepsilon \\
0 & \ddots & 0 & \vdots \\
0 & 0 & \varepsilon_{k-1, k-1} r & \left(M_{k-1}\right) \varepsilon \\
\hline & \mathbf{0} & & \varepsilon R \varepsilon
\end{array}\right]: r \in \mathbb{Z}\right\},
$$

and this embeds $R^{\prime}$ as a subring of the block matrix ring whose diagonal blocks are $k-1$ copies of $\varepsilon^{\prime} R \varepsilon^{\prime}$ and $\varepsilon R \varepsilon$. Since $\varepsilon R \varepsilon$ is quasi-Frobenius by hypothesis, it is also isomorphic to a matrix ring over $\varepsilon^{\prime} R \varepsilon^{\prime}$, so $R^{\prime}$ embeds in $\mathfrak{B}=\mathcal{M}_{k}\left(\varepsilon^{\prime} R \varepsilon^{\prime}\right)$ and we give this matrix ring the same $k \times k$ block structure as $R^{\prime}$. Since the entire last column $\mathfrak{B} \varepsilon$ of $\mathfrak{B}$ is contained in $R^{\prime}$ and $R^{\prime} \varepsilon$ is essential in $R^{\prime}$, for any submodule 
$M \subseteq \mathfrak{B} / R^{\prime}, \operatorname{hom}_{R^{\prime}}\left(M, R^{\prime}\right)=0$. Thus, the quasi-Frobenius ring $\mathfrak{B}=\mathcal{M}_{k}\left(\varepsilon^{\prime} R \varepsilon^{\prime}\right)$ is a rational extension of $R^{\prime}$. $\mathfrak{B}_{R^{\prime}}$ must then embed in $E\left(R^{\prime}\right)$ and contain the socle of $R^{\prime}$. By [9], the maximum rational extension $Q\left(R^{\prime}\right)$ of the ring $R^{\prime}$ is the double centralizer of $E\left(R^{\prime}\right)$, that is, $Q\left(R^{\prime}\right) \cong \operatorname{End}_{\Lambda}\left({ }_{\Lambda} E\left(R^{\prime}\right)\right)$ under the map $\operatorname{End}_{\Lambda}\left({ }_{\Lambda} E\left(R^{\prime}\right)\right) \ni \mu \mapsto(1) \mu \in$ $Q\left(R^{\prime}\right)$, so $E\left(R^{\prime}\right)$ is a right $Q\left(R^{\prime}\right)$-, and hence, an $\mathcal{M}_{k}(A)$-module. Since $E\left(R^{\prime}\right)$ is essential over $\mathcal{M}_{k}\left(\varepsilon^{\prime} R \varepsilon^{\prime}\right)$ as an $\mathcal{M}_{k}\left(\varepsilon^{\prime} R \varepsilon^{\prime}\right)$-module and $\mathcal{M}_{k}\left(\varepsilon^{\prime} R \varepsilon^{\prime}\right)_{\mathcal{M}_{k}\left(\varepsilon^{\prime} R \varepsilon^{\prime}\right)}$ is injective, $E\left(R_{R^{\prime}}^{\prime}\right)=\mathcal{M}_{k}\left(\varepsilon^{\prime} R \varepsilon^{\prime}\right)$. Since $R$ is essential over $R^{\prime}$, we have that $R^{\prime}$ embeds in $R$ which embeds in $\mathcal{M}_{k}\left(\varepsilon^{\prime} R \varepsilon^{\prime}\right)$, so $\mathcal{M}_{k}\left(\varepsilon^{\prime} R \varepsilon^{\prime}\right)$ is also $E(R)$ and a rational extension of $R$.

$\operatorname{Re}($ ii) $\Longrightarrow$ (i): (ii) is a special case of (i).

$\operatorname{Re}(\mathrm{i}) \Longrightarrow$ (iii): We initially proceed in a manner similar to the proof of Example 2. $\varepsilon E \cong \bigoplus_{i=1}^{k} E\left(\widetilde{S}_{i}\right)$, where

$$
k=\ell\left(\operatorname{socle}\left(\varepsilon R_{R}\right)\right)=\ell\left(\operatorname{socle}\left(\varepsilon E_{R}\right)\right)=\ell\left(\operatorname{socle}\left(\varepsilon E_{E}\right)\right)=\ell(\operatorname{socle}(\varepsilon E \varepsilon)) .
$$

Let $\varepsilon^{\prime \prime}$ be a primitive idempotent in $E$. Then $\varepsilon E \varepsilon \cong \operatorname{End}_{E}(\varepsilon E)$ is isomorphic to $k \times k$ matrices over the local ring $\varepsilon^{\prime \prime} E \varepsilon^{\prime \prime}$. By Theorem $6 E$ is quasi-Frobenius and so is the Morita equivalent ring $\varepsilon^{\prime \prime} E \varepsilon^{\prime \prime}$. Moreover, $\varepsilon^{\prime \prime} J(E) \varepsilon^{\prime \prime}$ must annihilate the socle of $\varepsilon^{\prime \prime} E \varepsilon^{\prime \prime}$. This says that socle $\left(\mathcal{M}_{k}\left(\varepsilon^{\prime \prime} E \varepsilon^{\prime \prime}\right)\right)$ is a $k^{2}$-dimensional vector space over top $\left(\varepsilon^{\prime \prime} E \varepsilon^{\prime \prime}\right)$, and this socle is an essential extension as an $R$-module of the length $k$ right socle of $\varepsilon R \varepsilon$. Since the top of $\varepsilon R \varepsilon$ is a simple artinian ring it must be the ring of $m \times m$ matrices over the division ring $\Delta=\operatorname{top}\left(\widetilde{\varepsilon}_{i} R\right)$ for any primitive idempotent $\widetilde{\varepsilon}_{i} \in R$. Then $\widetilde{S}$ must be $m$-dimensional over $\Delta$ on both sides. Hence, $m k=k^{2}$, so $m=k$ and the top of $\varepsilon R \varepsilon$ is bimodule-isomorphic to the socle of $\varepsilon R \varepsilon$, so $\varepsilon R \varepsilon$ is quasi-Frobenius.

We note that this also implies that $\varepsilon^{\prime}$ remains primitive in $E$, and every indecomposable projective $E$-module is isomorphic to $\varepsilon^{\prime} E$ and must contain an $R$ submodule $M \cong \varepsilon^{\prime} R$.

By (the proof of) (iii) $\Longrightarrow$ (ii), the subring of $E$ generated by $\varepsilon R \varepsilon$ and, for each $i<k$, an $M_{i} \cong \varepsilon^{\prime} R$ contained in $E\left(S_{i}\right)$ embeds $E$ as a rational submodule of $\mathcal{M}_{k}\left(\varepsilon^{\prime} R \varepsilon^{\prime}\right)$ and this makes $E \cong \mathcal{M}_{k}\left(\varepsilon^{\prime} R \varepsilon^{\prime}\right)$. Set $N_{i}=M_{i} \cap R$ for $i<k$. Now look at the $2 \times 2$ upper triangular submatrix $\left[\begin{array}{cc}\varepsilon_{i} E \varepsilon_{i} & N_{i} \\ 0 & \varepsilon R \varepsilon\end{array}\right]$ whose 1,1 entry is the $i, i$ entry of $E$ and whose second column is contained in $R$. Multiplying the top row by the second column gives $\left[\varepsilon_{i} E \varepsilon_{i} N_{i} \varepsilon^{\prime} N_{i} \varepsilon^{\prime}\right] R \subseteq E\left(\widetilde{S}_{i}\right)$. Thus, the right $R$-module corresponding to the top row will have a simple $R$-module $\widetilde{S}_{i}$ contained in the last column, and if $N_{i} \varepsilon^{\prime} \subseteq J\left(\operatorname{hom}_{E}\left(E\left(\varepsilon^{\prime} \widetilde{S}_{k}\right), E\left(\widetilde{S}_{i}\right)\right)\right)$, it will also have a simple $R$-module isomorphic to $\widetilde{S}$ in the 1,1 position (or $i, i$ position of $E$ looked at as a matrix ring). But row $i$ of $E$ is $E\left(\widetilde{S}_{i}\right)$ which has a length 1 socle, so $N_{i}=M_{i} \subseteq R$.

In our next result, we show that the set theoretic uniqueness of ring structures on $E$ is equivalent to the set theoretic uniqueness of injective hulls if $R$ is Osofsky compatible and $E_{R}$ has finite length. Theorem 8 plays a significant role in the proof of this theorem.

THEOREM 9. Let $E_{R}$ have finite length. Then the following are equivalent.

(i) E has a set theoretically unique compatible ring structure.

(ii) $E$ has a compatible ring structure as a direct product $\prod R_{i}$ of rings $R_{i}$ of two types: either every primitive idempotent of $R_{i}$ remains primitive in $E$ or there is only one isomorphism class of simple modules in the socle of $R_{i}$.

(iii) $E_{R}$ is a rational extension of $R_{R}$. 
Proof. It is enough to prove this if $R$ is basic, since the proof in the basic case will go through for the general case by simply replacing isomorphism classes of principal indecomposable projectives by sums of the members of the isomorphism class which are matrices over the endomorphism ring of one of the indecomposable projectives. This is precisely the relationship between $\varepsilon^{\prime} R \varepsilon^{\prime}$ and $\varepsilon R \varepsilon$ in Theorem 8 . Of course, one cannot assume that $E$ is basic as at least one simple will occur with multiplicity greater than 1 in the socle of $R$ unless $R$ is quasi-Frobenius.

(iii) implies (i). This is well known, and indeed one of the first results mentioned in this paper.

(i) implies (ii). If $E$ does not have a compatible ring structure, there is nothing to prove. So now assume it does have a compatible ring structure $E_{\lambda}$ which we will denote just by $E$.

Now let $e=e^{2} \in E \backslash R, e$ primitive. Let $S_{E}=e E / e J(E)$, the top of $e E$, and assume that $S$ is not isomorphic to $\operatorname{socle}\left(e E_{R}\right)$. Since $S$ embeds in the socle of $E, S$ must be isomorphic to the socle of some $e^{\prime} E$ such that the top of $e^{\prime} E$ is not isomorphic to $S$. By Proposition 3 there is a set theoretically different ring structure on $E$ for which $e$ is not idempotent. This contradiction shows that the socle of $e E$ must be isomorphic to $S$. Then the dual of $S=\operatorname{socle}(e E)$ will equal the socle of $E e$. (See Proposition 2.)

Let us recall a definition given in the proof of Proposition 2. We denoted by $\varepsilon_{S}$ the idempotent $\varepsilon_{S}=\varepsilon_{S}^{2} \in E$ which is central modulo $J(E)$ and has only one isomorphism class of simple modules, namely [S]. Then $\varepsilon_{S} E \varepsilon_{S} \cong \operatorname{hom}_{E}\left(\varepsilon_{S} E, \varepsilon_{S} E\right)$ is a subring of $E$ whose socle is the homogeneous component $H_{S}$ of the socle of $E$. All right composition factors of $\varepsilon_{S} E \varepsilon_{S}$ are isomorphic to $S$ and their duals are isomorphic to the dual of $S$, so all left composition factors are isomorphic to the dual of $S$. The dual of $S, E e$, and its dual $S$ are simple in $E$ and hence in $\varepsilon_{S} E \varepsilon_{S}$, so $\varepsilon_{S} E \varepsilon_{S}$ is quasi-Frobenius. For all other simple modules $S^{\prime}$ in the socle of $E$ but not in $H_{S}$, any homomorphism in either direction between a composition factor of $\varepsilon_{S} E \varepsilon_{S}$ and $S^{\prime}$ is 0 . That says $\varepsilon_{S} E \varepsilon_{S}$ is a ring direct factor of $E$. By induction we get

$$
E \supseteq\left(\prod_{\substack{\exists e=e^{2} \in E \backslash R \\ S \cong e E / e J(E)}} \varepsilon_{S} E \varepsilon_{S}\right) \times \mathcal{C}=\Re,
$$

where $\mathcal{C}$ is the injective hull of the sum of the remaining isomorphism classes of simples in the socle of $E$.

Every simple not in $\mathcal{C}$ is already mapped to itself by the one-to-one correspondence between isomorphism classes of simples in the tops of indecomposable projectives of $E$ and isomorphism classes of simples in the socle, so simple tops in $\mathcal{C}$ also appear as simples in the socle of $\mathcal{C}$. Since all composition factors of $\mathcal{C}$ are isomorphic to simples in the socle of $\mathcal{C}$ and in the top of $\mathcal{C}$, if we set $\varepsilon_{C}=1-\sum_{\{S \cong e E / e J(E): e \notin R\}} \varepsilon_{S}$, we get exactly as for $\varepsilon_{S} E \varepsilon_{S}$ that duals of simple $\mathcal{C}=\varepsilon_{C} E \varepsilon_{\mathcal{C}}$-modules are simple, so $\mathcal{C}$ is a quasi-Frobenius ring.

Since every primitive idempotent in $E \backslash R$ lies in some $\varepsilon_{S} E \varepsilon_{S}$, the primitive idempotents in $\mathcal{C}$ must lie in $R$.

The ring $\Re$ in $(*)$ is thus a quasi-Frobenius subring of $E$ which contains an essential $R$-submodule of $E$ and, because it maps isomorphically onto $R / J(R)$ it also contains the identity of $R$. Then $E$ cannot split as $\mathfrak{R}_{R} \oplus M_{R}$ unless $M=0$. Thus, $E=\Re$. 
(ii) implies (iii). We observe that the injective hull of a direct product of rings is the direct product of the injective hulls of the factors, and there are no non-zero maps between the injective hulls of the factors, so the extension is rational if and only if the injective hulls of the factors are rational over the factor rings. So we look at the two kinds of rings separately.

If $R=\varepsilon_{S} E \varepsilon_{S}$, this is part of Theorem 8 .

Let $E=\mathcal{C}$. Let $\mathfrak{P}$ denote a set of orthogonal primitive idempotents which sum to 1 . For each simple $S_{i}$ in the socle of $R$, there is a subset $\mathfrak{P}_{i} \subseteq \mathfrak{P}$ such that the $S_{i}$-homogeneous component of the socle of $R$ is the socle of $\sum_{e_{j} \in \mathfrak{P}_{i}} e_{j} R$. Now look at the right ideal $C_{i}=\sum_{e_{j} \in \mathfrak{P}_{i}} e_{j} E$ of $E . E / J(E)$ is a product of simple rings, and each of the factors arises as the top of precisely one of the $C_{i}$. In the notation in Proposition 2, the simple module $S_{\sigma^{-1}(i)}$ in the top of $C_{i}$ is an $R$-module with

$$
\ell\left(\left(S_{\sigma^{-1}(i)}\right)_{R}\right)=\left|\mathfrak{P}_{i}\right|=\ell\left(\operatorname{socle}\left(C_{i}\right)\right),
$$

so each idempotent of $\mathfrak{P}_{i}$ contributes a distinct $R$-composition factor of $S_{\sigma^{-1}(i)}$, so there can be no non-zero maps from the $R$-module $S_{\sigma^{-1}(i)}$ to the $R$-module $S_{\sigma^{-1}(i)} /$ socle $\left(S_{\sigma^{-1}(i)}\right)$. Then $(E / J(E))_{R}$ is a rational extension of its socle.

Now let $\mu \in \mathfrak{I}=\{\lambda \in \Lambda: \lambda(1)=0\}$. Then $\operatorname{ker}(\mu)+J(E)=E$, and since $J(E)$ is contained in the Jacobson radical of the $\operatorname{ring} R+J(E)$ it will be small in $E$, so $\operatorname{ker}(\mu)=E$ and $\mathfrak{I}=0$, and thus, $E$ is a rational extension of $R$ by Proposition 1 .

It need not be true that all primitive idempotents of $E$ lie in $R$ if $E$ is a rational extension of $R_{R}$, as shown in Example 3.

4. Concluding remarks. These results show that compatible ring structures on $E$, if they exist, behave very much like the injective module $E$ itself if we have finite length. Set theoretically distinct injective hulls correspond to set theoretically distinct compatible ring structures, although all compatible ring structures are isomorphic. And $E$ is a rational extension of $R$ if and only if the ring structure is set theoretically unique. By Theorem 9, we know precisely what an Osofsky compatible ring with $E_{R}$ of finite length must look like. And we have a complete characterization of Osofsky compatible rings with only one isomorphism class of simples in its socle by Theorem 8 .

To help understand Theorem 8, we can apply it to several examples in Section 2. For Example 2, the only primitive idempotent is 1 , and $R$ is not essential over any simple in its socle, so $R$ is not Osofsky compatible. For Example 5, $\left[\begin{array}{cc}0 & 2 \mathbb{Z}_{4} \\ 0 & 0\end{array}\right]$ does not have an essential extension in $R$ isomorphic to the right ideal $\left[\begin{array}{ll}0 & 0 \\ 0 & \mathbb{Z}_{4}\end{array}\right]$ so it cannot be Osofsky compatible. For Example 4 the sum in Theorem 8 is not a problem, but the ring is not quasi-Frobenius. If one modifies that example to look at the ring $\left[\begin{array}{cc}K_{K}{ }_{K} K_{F} \\ 0\end{array}\right]$ the hypotheses in Theorem 8 hold, and indeed the injective hull of this new ring is a rational extension of it as Proposition 5 shows. For Example 7, Theorems 8 and 9 do not apply directly, but you can observe that the hypotheses of Theorem 8 hold on each homogeneous component of the socle individually, and this might or might not give some insight into the non-rational case.

There is one wide open question remaining on compatible ring structures on injective hulls of artinian rings, or perhaps just Artin algebras if one wishes to eliminate examples such as Example 4. The problem is to find 'nice' conditions equivalent to the 
existence of a compatible ring structure in the absence of a rational extension. We have some significant clues from the structure of $E / J(E)_{R}$ developed to prove Theorem 9. Being able to get a $\Lambda / J(\Lambda)-\Lambda / J(\Lambda)$ bimodule structure on the right annihilator of the left socle of ${ }_{\Lambda} E$ is a necessary condition for having a compatible ring structure. But is it sufficient together with some of the necessary conditions shown above? What conditions on the artinian ring $R$, with $E_{R}$ of finite length, are equivalent to $R$ being Osofsky compatible if $R$ has at least two non-isomorphic simples appearing in its socle and some principal indecomposable projective with non-simple socle? At this time, we have no serious conjecture as to what necessary and sufficient conditions might look like.

ACKNOWLEDGEMENTS. The second and third authors are grateful for the support they received from the Mathematics Research Institute, Columbus, Ohio, Busan National University, South Korea, and the Ohio State University at Lima.

\section{REFERENCES}

1. F. W. Anderson and K. R. Fuller, Rings and categories of modules, second ed., Graduate Texts in Mathematics, vol. 13 (Springer-Verlag, New York, 1992).

2. G. F. Birkenmeier, B. L. Osofsky, J. K. Park and S. T. Rizvi, Injective hulls with distinct ring structures, J. Pure Appl. Algebra 213 (2009), 732-736.

3. G. F. Birkenmeier, J. K. Park and S. T. Rizvi, An essential extension with nonisomorphic ring structures, Algebra and Its Applications, Contemp. Math., vol. 419 (Amer. Math. Soc., Providence, RI, 2006), 29-48.

4. G. F. Birkenmeier, J. K. Park and S. T. Rizvi, An example of Osofsky and essential overrings, Rings, modules, and representations, Contemp. Math, vol. 480 (Amer. Math. Soc., Providence, RI, 2009), 12-33.

5. V. Camillo, I. Herzog and P. P. Nielsen, Non-self-injective injective hulls with compatible multiplication, J. Algebra 314(1) (2007), 471-478. $1223-1227$

6. F. Dischinger and W. Müller, Left PF is not right PF, Comm. Algebra 14(7) (1986),

7. C. Faith, Lectures on injective modules and quotient rings, Lecture Notes in Mathematics, No. 49 (Springer-Verlag, Berlin, 1967).

8. C. Faith and Y. Utumi, Maximal quotient rings, Proc. Amer. Math. Soc. 16 (1965), $1084-1089$.

9. G. D. Findlay and J. Lambek, A generalized ring of quotients. I, II, Can. Math. Bull. 1 (1958), 77-85, 155-167.

10. R. E. Johnson, Quotient rings of rings with zero singular ideal, Pacific J. Math. 11 (1961), 1385-1392.

11. T. Y. Lam, Lectures on modules and rings, Graduate Texts in Mathematics, vol. 189 (Springer-Verlag, New York, 1999).

12. N. C. Lang, On ring properties of injective hulls, Can. Math. Bull. 18(2) (1975), 233-239.

13. K. Morita, Duality for modules and its applications to the theory of rings with minimum condition, Sci. Rep. Tokyo Kyoiku Daigaku A 6 (1958), 83-142.

14. B. L. Osofsky, Homological properties of rings and modules, Doctoral Dissertation (Rutgers University, New Brunswick, NJ, 1964).

15. B. L. Osofsky, On ring properties of injective hulls, Can. Math. Bull. 7 (1964), 405-413.

16. B. L. Osofsky, A non-trivial ring with non-rational injective hull, Can. Math. Bull. 10 (1967), 275-282.

17. B. L. Osofsky, Endomorphism rings of quasi-injective modules, Can. J. Math. 20 (1968), 895-903. 